\section{Tendência e desigualdade na completude da informação sobre raça/cor dos óbitos de idosos no Sistema de Informações sobre Mortalidade no Brasil, entre 2000 e 2015}

\author{
Trend and inequality in the completeness of \\ information on race/color in deaths of elderly \\ in the Mortality Information System in Brazil, \\ 2000 to 2015
}

Tendencia y desigualdad en la cumplimentación de la información sobre raza/color de los óbitos de ancianos en el Sistema de Informacions sobre Mortalidad en Brasil, entre 2000 y 2015

\section{Resumo}

A qualidade da informação sobre raça/cor é condição necessária para conhecer o impacto da desigualdade na mortalidade. O objetivo deste trabalho é analisar a tendência e a desigualdade na completude da raça/cor dos óbitos de idosos no Sistema de Informações sobre Mortalidade (SIM) entre 2000 e 2015 no Brasil. Analisa-se a completude dessa variável por diferentes abrangências geográficas, a raça/cor mais afetada pela má completude e a associação entre a excelência do preenchimento da raça/cor com a territorialidade e a condição socioeconômica dos municípios. Os dados dos óbitos de idosos provêm do SIM, e as informações de população, dos Censos e estimativas do Ministério da Saúde. Estima-se a variação percentual da proporção da incompletude. A variação percentual de pretos/pardos foi estimada entre 2000 e 2010 para o SIM e Censos. Estima-se regressão logística simples e ajustada (IC95\%), tendo, como desfecho excelente, completude da raça/cor e, como resposta, variáveis territoriais e socioeconômicas. Encontrou-se acentuada melhora da qualidade do preenchimento no período, especialmente até 2006, sendo excelente a média nacional desde 2007. Nota-se desigualdade territorial em nível municipal. Municípios de IDH baixo/médio, com alta proporção de pobreza e desigualdade tiveram menor chance de ter excelente completude. O modelo ajustado mostra que a região e o tamanho do município são as características que explicam a excelente qualidade da variável raça/cor. Municípios do Nordeste $e$ de pequeno porte têm menos chance de excelente completude. Conclui-se que raça/cor no SIM tem qualidade para ser utilizada nos estudos de desigualdade da mortalidade dos idosos, salvo exceções em nível municipal.

Idoso; Registros de Mortalidade; Confiabilidade dos Dados;

Fatores Raciais; Racismo
Dalia Elena Romero 1

Leo Maia 1

Jessica Muzy ${ }^{1}$

doi: 10.1590/0102-311X00223218

\section{Correspondência}

D. E. Romero

Rua Cosme Velho 318, bloco 2, Rio de Janeiro, RJ

22241-090, Brasil.

dalia.fiocruz@gmail.com

1 Instituto de Comunicação e Informação Científica e Tecnológica em Saúde, Fundação Oswaldo Cruz, Rio de Janeiro, Brasil. 


\section{Introdução}

A raça/cor é um construto social 1,2. Evidências científicas mostram que as variações da cor da pele e do fenótipo não implicam diferenças biológicas ou genéticas que justifiquem a diferenciação dos indivíduos ${ }^{3}$. Entretanto, pelo legado histórico de discriminação e exploração, a cor da pele passou a constituir um determinante socioeconômico 4 e uma condição objetiva de desigualdade nas condições de vida, saúde e morte 2,5,6.

No Brasil, embora haja uma escassez de análises sobre o tema 2,7, estudos têm mostrado piores condições de saúde da população negra (pretos e pardos) em relação aos brancos, o que leva ao maior risco de mortalidade infantil 8 , por causas externas 9,10,11, por complicações na gravidez e no parto 11 e por transtornos mentais 10,11. Desvantagens na qualidade da informação das causas de óbitos para essa população também têm sido demonstradas 10,11,12.

Para a população idosa, ainda que em menor número, também existem evidências de desigualdade na saúde segundo raça/cor. Em estudo realizado com base na Pesquisa Nacional por Amostra de Domicílios (PNAD) de 2008, verifica-se que idosos negros têm pior autoavaliação de saúde em relação aos brancos 13. Já sobre a desigualdade na mortalidade dos idosos segundo raça/cor, pouco se conhece, possivelmente por conta dos problemas observados durante décadas na qualidade das informações socioeconômicas nas estatísticas vitais no Brasil 14. No pouco que foi estudado, verificou-se que idosos brancos atingem idades mais longevas em relação aos demais 15 .

A relevância da informação sobre raça/cor para a avaliação das políticas de saúde é reconhecida pelo Estado brasileiro. A recente Política Nacional de Informação e Informática em Saúde (PNIIS) 16 aponta a informação em saúde como elemento estruturante para o fortalecimento do SUS e a garantia dos direitos de cidadania e identifica a informação de raça/cor como central na eliminação das desigualdades. Seu uso é importante para a avaliação do desempenho do SUS, o qual tem, como um de seus princípios, a equidade em saúde e considera a desigualdade social como injusta e evitável 17.

A Lei Orgânica da Saúde (Lei no 8.080/1990) prevê, como competência e atribuição comum à União, aos Estados e Municípios, a organização e coordenação do Sistema Nacional de Informação em Saúde (SNIS). A PNIIS-2016 direciona-se para o acesso e a qualidade da informação em saúde, em sintonia com as necessidades decorrentes da heterogeneidade e desigualdade das regiões e municípios brasileiros. Um estudo sobre adequação das informações de nascimentos e óbitos do Ministério da Saúde mostrou que as desigualdades ocorrem tanto por porte populacional como por municípios e macrorregião geográfica 18 .

Sabe-se que as estatísticas vitais, em virtude de seu caráter contínuo e amplitude nacional, assim como pela disponibilidade de relevantes variáveis socioeconômicas e demográficas, constituem fontes de informação privilegiadas para o estudo e monitoramento da desigualdade em saúde 14. O Sistema de Informações sobre Mortalidade (SIM), estabelecido em 1975 pelo Ministério da Saúde, tem como instrumento de coleta a Declaração de Óbito (DO). A DO tem experimentado algumas modificações, mas o quesito raça/cor está presente de forma contínua desde 199619 e mantém as mesmas categorias de resposta: branca, preta, amarela, parda e indígena.

No último manual da DO 20, o Ministério da Saúde reafirmou a relevância do quesito raça/cor na identificação de agravos que acometem a população em situação de vulnerabilidade, como negros e indígenas, e aponta que a não consideração da composição pluriétnica da sociedade brasileira implicaria impedir sistematicamente a equidade na atenção à saúde.

A qualidade da informação socioeconômica nos sistemas de informação é condição necessária para conhecer, de forma fidedigna, o impacto da desigualdade na mortalidade. Assim, a Política Nacional de Saúde Integral da População Negra (PNSIPN) do Ministério da Saúde 21 estabelece como um de seus objetivos específicos a melhora na qualidade dos sistemas de informação do SUS no que tange à coleta, ao processamento e à análise dos dados desagregados por raça, cor e etnia, além da inclusão do quesito cor em todos os instrumentos de coleta de dados, especialmente os relativos à morbidade e à mortalidade. Existe também uma recente Portaria do Ministério da Saúde 22 que dispõe sobre ações para que formulários dos sistemas de informação em saúde, bem como as informações epidemiológicas divulgadas anualmente pelo SUS contenham dados de boa qualidade sobre raça/cor.

Apesar dessas demandas, os poucos estudos que avaliam a qualidade da informação da variável raça/cor e mostram melhoras no preenchimento limitam a análise até 2011, concentram-se quase que 
exclusivamente na mortalidade infantil 14,23 e em específicas abrangências, como Vitória 24 , Pernambuco 25,26 , Bahia 27 , Ceará 28 e Região Sudeste 29 , e em óbitos de grupos de causas específicas, como externas 25,28 , câncer de mama 29 e suicídio 27,30 .

No que tange à população idosa, os estudos sobre a qualidade da variável raça/cor no SIM são raros 27,28 . Os poucos artigos produzidos apenas consideram a mortalidade por causas específicas e não têm representatividade nacional 27,28. Conhecer o grau de qualidade dessa informação é fundamental para estabelecer a potencialidade do SIM para estudar a desigualdade da mortalidade no processo de envelhecimento, especialmente com as acentuadas mudanças demográficas e epidemiológicas observadas nas recentes décadas 31 . Estudar os diferenciais raciais ante a morte é uma questão essencial para orientar políticas de saúde para prevenção de agravos, especialmente ao levar em conta a maior longevidade da população.

Existem evidências da menor completude no preenchimento das variáveis socioeconômicas entre os mais pobres $14 \mathrm{e}$ da menor cobertura do SIM em áreas mais desfavorecidas 32 . Sabe-se que existem amplas desvantagens dos pretos e pardos em relação aos brancos na qualidade de vida e saúde 33 . Com isso, este trabalho tem como hipótese que, no caso de melhora da completude da variável raça no SIM, aumente a proporção de pretos e pardos entre os óbitos dos idosos, e sua estrutura assemelhe-se gradualmente à composição observada na população geral.

O principal objetivo deste trabalho é analisar a tendência e a desigualdade na completude da variável raça/cor dos óbitos de idosos no SIM entre 2000 e 2015. Para tal, analisa-se a completude dessa variável de 2000 a 2015 , segundo diferentes abrangências geográficas, identifica-se a raça/cor mais afetada pela má completude e verifica-se a associação entre a excelência do preenchimento da raça/ cor com a territorialidade e a condição socioeconômica dos municípios.

\section{Metodologia}

\section{Fontes de dados e variáveis utilizadas}

As informações sobre mortalidade utilizadas neste trabalho são oriundas das declarações de óbito de idosos a partir de 60 anos de idade, registradas no SIM, no período entre 2000 e 2015. Os microdados foram obtidos a partir do Departamento de Informática do SUS (DATASUS) do Ministério da Saúde. Dessa fonte, foram utilizadas as variáveis de raça/cor, região, Unidade da Federação (UF) e município. O tamanho dos municípios (número de habitantes) foi calculado a partir de estimativas preliminares de população elaboradas pelo Coordenação-Geral de Informações e Análises Epidemiológicas, Secretaria de Vigilância em Saúde, Ministério da Saúde (CGIAE/SVS). Dados sobre população segundo raça/cor e pobreza são oriundos dos Censos Demográficos de 2000 e 2010.

A definição de pobreza utilizada neste estudo é a mesma do Censo, que se refere a indivíduos que têm renda domiciliar per capita de até $1 / 2$ salário mínimo. Os indicadores de desigualdade, tais como Índice de Desenvolvimento Humano (IDH) e Índice de Gini foram obtidos pelo Atlas do Desenvolvimento Humano 2010 34. O IDH é calculado a partir de informações sobre longevidade, educação e renda; e o Gini representa o afastamento de uma dada distribuição de renda. O Censo, por ser fonte robusta e de caráter universal, também foi utilizado para comparar a composição da raça/cor da população idosa com a composição dos óbitos dessa no SIM.

A informação de raça cor, segundo o manual de preenchimento da DO 20, deve ser reportada ao médico pelo responsável do falecido, o qual deve ser informado das opções de resposta da variável, sendo elas: (1) Branca; (2) Preta; (3) Amarela (pessoa de origem oriental: japonesa, chinesa e coreana, entre outras); (4) Parda (inclui-se, nessa categoria, morena, mulata, cabocla, cafuza ou qualquer outro mestiço de pessoa da cor preta com pessoa de outra cor ou raça); (5) Indígena (se aplica aos indígenas ou índios que vivem em aldeamento, como também aos que se declararam indígenas e vivem fora do aldeamento). O registro de todas as informações na DO é de responsabilidade do médico e cabe a ele zelar pela sua qualidade.

O indicador de incompletude é definido como a proporção de informação com campos em branco na DO, segundo diversos autores $35,36,37$. A informação de raça/cor não aceita resposta ignorada na DO, como especificado no manual 20. A proporção de incompletude é estimada segundo regiões, UF 
e tamanho dos municípios. Utiliza-se o escore proposto por Romero \& Cunha 14 com os seguintes graus de avaliação: excelente (menor de 5\%), bom (5\% a 10\%), regular (10\% a 20\%), ruim (20\% a 50\%) e muito ruim (50\% ou mais).

\section{Análise estatística}

Estimou-se a variação percentual da proporção de incompletude. Como numerador, utilizou-se a diferença do percentual de incompletude entre o último e o primeiro ano analisados (2015 e 2000, respectivamente), e, como denominador, o percentual de incompletude do primeiro ano. Também foi estimada a variação percentual da proporção de pretos e pardos entre os anos 2000 e 2010, tanto no Censo como no SIM, seguindo o mesmo método de cálculo.

A proporção de incompletude do preenchimento da variável raça/cor foi calculada para regiões e UF de 2000 a 2015. Com a finalidade de analisar a desigualdade territorial da qualidade da informação de raça/cor, calculou-se a amplitude dos valores observados para a proporção de incompletude ano a ano, subtraindo o menor valor do maior encontrado entre as UF. Para a análise em nível municipal, calculou-se a proporção de municípios com excelente preenchimento $(<5 \%$ de incompletude) entre 2000 e 2015, com intervalos de cinco anos, segundo regiões e UF.

Para analisar o efeito da melhora da completude na distribuição dos óbitos por raça/cor, foi realizada uma comparação dos anos 2000 e 2010 quanto à proporção dos óbitos de pretos e pardos de idosos no SIM com a proporção de pretos e pardos da população idosa nos respectivos Censos. Estima-se a variação percentual entre 2000 e 2010 na estrutura por cor de cada uma das fontes de informação assim como a razão entre elas a partir da metodologia de cálculo da variação percentual supracitada.

Para investigar a relação entre a qualidade do preenchimento da variável raça/cor no SIM e a territorialidade e desigualdade socioeconômica nos municípios, calcula-se regressão logística simples e ajustada, com nível de $95 \%$ de confiança. A variável desfecho foi definida como excelente completude (> 95\%) da raça/cor. Como variáveis resposta, selecionaram-se características dos municípios relacionados com a condição territorial (região e tamanho do município); condição socioeconômica no município (Índice de Gini e Proporção de Pobreza) e IDH municipal.

\section{Resultados}

Foi observada uma melhora da completude da variável raça/cor dos óbitos de idosos desde o ano 2000. Nesse ano, mais da metade das UF (16) já tinham excelente ou bom preenchimento (menos de $10 \%$ de incompletude). A Região Nordeste era a única que não alcançava boa completude nesse ano. Entretanto, dez anos depois, apenas duas UF dessa mesma região, Alagoas e Rio Grande do Norte, ainda não tinham alcançado esse patamar. A partir de 2012, todas as UF apresentavam bom ou excelente preenchimento (Tabela 1).

A redução da incompletude da variável raça/cor entre os anos 2000 e 2015 foi de mais de 50\%, evidenciada pela variação percentual, em quase todas as regiões e UF. Apenas Tocantins, Rio Grande do Norte, Espírito Santo e Rio Grande do Sul, as quais, com exceção da última, já estavam próximas de ter boa qualidade do preenchimento no começo da década, não variaram tanto. As regiões que apresentaram maior redução da incompletude foram Norte (variação percentual = -87,6\%), Nordeste (variação percentual = -73,9\%) e Centro-oeste (variação percentual = -76,8\%) (Tabela 1). Algumas UF que já possuíam excelente completude no ano 2000 também apresentaram melhora e quase alcançaram a totalidade da completude.

A desigualdade na qualidade da informação sobre raça/cor dos idosos nas UF apresentou redução no período. Em 2000, a diferença entre o valor máximo e mínimo de incompletude encontrada era de 26,9 pontos percentuais. Até 2012, essa amplitude diminui progressivamente e chega a 8,1 em 2015. Assim, entre 2000 e 2015, houve uma redução de quase $70 \%$ na diferença de valores entre as UF. Nesse mesmo ano, verificou-se que as UF com percentuais mais elevados de incompletude conseguiram igualar-se às que já apresentavam valores baixos no ano 2000 (Tabela 1).

Ao analisar uma escala territorial menor, nota-se que, do total de municípios (5.591), a maioria chega a 2015 com excelente preenchimento, que passa de 41,8\%, em 2000, para 71\%, em 2015 (Tabela 2). 


\section{Tabela 1}

Percentual de incompletude da variável raça/cor, variação percentual entre primeiro e último anos e número de óbitos de idosos para regiões, Unidades da Federação (UF) e Brasil, 2000-2015.

\begin{tabular}{|c|c|c|c|c|c|c|c|c|c|c|c|c|c|c|c|c|c|c|}
\hline \multirow[t]{2}{*}{ UF } & \multicolumn{16}{|c|}{$\%$ incompletude } & \multirow{2}{*}{$\begin{array}{c}\text { Variação } \\
\text { percentual } \\
\text { 2000-2015 }\end{array}$} & \multirow{2}{*}{$\begin{array}{c}\text { Número } \\
\text { de óbitos } \\
2015\end{array}$} \\
\hline & 2000 & 2001 & 2002 & 2003 & 2004 & 2005 & 2006 & 2007 & 2008 & 2009 & 2010 & 2011 & 2012 & 2013 & 2014 & 2015 & & \\
\hline Norte & 8,4 & 6,6 & 2,8 & 2,4 & 2,4 & 2,1 & 2,3 & 2,2 & 2,2 & 1,8 & 1,5 & 1,4 & 1,2 & 1,2 & 1,2 & 1,1 & $-87,6$ & 41.342 \\
\hline RO & 9,3 & 5,7 & 6,8 & 6,3 & 7,0 & 3,7 & 3,3 & 6,2 & 5,6 & 2,7 & 2,0 & 1,8 & 1,8 & 1,4 & 1,7 & 1,4 & $-84,7$ & 4.438 \\
\hline$A C$ & 14,7 & 11,1 & 10,6 & 10,3 & 11,2 & 12,5 & 14,0 & 13,8 & 15,5 & 13,7 & 9,3 & 8,9 & 4,6 & 4,2 & 2,3 & 1,7 & $-88,6$ & 1.904 \\
\hline AM & 1,9 & 1,6 & 2,2 & 2,2 & 2,1 & 2,4 & 2,4 & 1,9 & 1,6 & 1,3 & 1,3 & 1,3 & 1,0 & 0,8 & 0,9 & 0,8 & $-56,3$ & 8.421 \\
\hline $\mathrm{RR}$ & 4,1 & 5,3 & 5,5 & 6,0 & 5,2 & 5,4 & 4,1 & 1,1 & 0,7 & 1,2 & 2,4 & 1,8 & 1,5 & 1,4 & 1,5 & 1,5 & $-63,0$ & 948 \\
\hline PA & 12,6 & 10,1 & 1,7 & 1,0 & 0,6 & 0,8 & 1,2 & 0,7 & 0,8 & 0,8 & 0,6 & 0,6 & 0,6 & 1,0 & 0,9 & 0,8 & $-93,9$ & 19.997 \\
\hline$A P$ & 3,3 & 1,7 & 1,3 & 0,2 & 1,0 & 0,1 & 1,0 & 1,0 & 2,1 & 0,8 & 2,3 & 1,5 & 1,0 & 1,8 & 1,3 & 1,5 & $-55,2$ & 1.350 \\
\hline TO & 1,8 & 1,9 & 0,9 & 0,9 & 1,1 & 0,6 & 0,6 & 0,8 & 1,0 & 1,6 & 1,7 & 1,7 & 2,3 & 1,7 & 2,2 & 2,0 & 7,6 & 4.284 \\
\hline Nordeste & 15,4 & 13,8 & 12,1 & 10,9 & 10,5 & 9,2 & 9,0 & 7,9 & 6,9 & 7,0 & 6,1 & 5,4 & 4,8 & 4,8 & 4,3 & 4,0 & $-73,9$ & 212.093 \\
\hline MA & 8,3 & 5,0 & 2,9 & 2,3 & 2,3 & 2,5 & 1,8 & 2,0 & 2,0 & 2,1 & 1,8 & 1,6 & 1,7 & 2,0 & 1,6 & 1,5 & $-81,6$ & 19.323 \\
\hline $\mathrm{PI}$ & 6,8 & 6,4 & 6,1 & 6,6 & 5,8 & 3,5 & 3,7 & 3,9 & 4,0 & 3,2 & 3,1 & 3,2 & 3,5 & 4,1 & 3,4 & 3,7 & $-46,2$ & 12.556 \\
\hline $\mathrm{CE}$ & 15,6 & 15,3 & 13,5 & 13,4 & 14,0 & 10,4 & 6,4 & 4,7 & 4,2 & 4,9 & 4,2 & 3,7 & 2,8 & 2,6 & 2,4 & 2,3 & $-85,3$ & 35.831 \\
\hline $\mathrm{RN}$ & 6,7 & 7,4 & 9,6 & 9,9 & 10,2 & 10,5 & 12,9 & 11,9 & 11,7 & 11,0 & 10,1 & 7,7 & 7,6 & 7,8 & 6,3 & 5,9 & $-11,6$ & 12.943 \\
\hline PB & 18,1 & 17,1 & 12,6 & 10,5 & 10,2 & 9,7 & 9,4 & 6,8 & 5,4 & 4,1 & 5,0 & 5,7 & 4,7 & 4,5 & 4,2 & 4,5 & $-75,0$ & 17.805 \\
\hline PE & 12,0 & 9,9 & 7,0 & 4,3 & 3,1 & 2,8 & 2,7 & 3,1 & 2,9 & 5,0 & 3,1 & 2,8 & 2,7 & 2,4 & 1,5 & 1,2 & $-89,7$ & 40.515 \\
\hline $\mathrm{AL}$ & 17,2 & 15,0 & 15,7 & 14,4 & 18,8 & 21,0 & 20,0 & 17,2 & 13,1 & 14,7 & 13,9 & 10,3 & 6,0 & 6,5 & 7,1 & 8,5 & $-50,6$ & 11.758 \\
\hline SE & 28,8 & 27,5 & 24,5 & 24,5 & 22,4 & 17,5 & 14,1 & 11,4 & 5,7 & 6,3 & 5,7 & 4,2 & 4,0 & 3,7 & 3,7 & 3,5 & $-88,0$ & 7.863 \\
\hline $\mathrm{BA}$ & 20,2 & 18,4 & 17,2 & 16,2 & 14,5 & 12,9 & 14,6 & 13,2 & 12,1 & 10,7 & 9,2 & 8,6 & 8,2 & 8,5 & 7,5 & 6,6 & $-67,3$ & 53.499 \\
\hline Sudeste & 6,4 & 5,5 & 5,0 & 4,2 & 3,6 & 3,4 & 4,7 & 4,5 & 4,3 & 4,2 & 4,0 & 3,6 & 3,5 & 3,4 & 3,1 & 2,9 & $-54,7$ & 391.945 \\
\hline MG & 13,4 & 11,8 & 10,9 & 11,0 & 10,0 & 9,2 & 8,9 & 8,5 & 8,3 & 8,0 & 7,2 & 6,3 & 6,0 & 7,0 & 6,8 & 6,4 & $-52,1$ & 88.040 \\
\hline ES & 15,2 & 14,7 & 14,2 & 13,8 & 12,6 & 13,6 & 13,9 & 14,0 & 14,5 & 12,9 & 9,0 & 9,5 & 7,8 & 7,5 & 8,0 & 8,0 & $-47,2$ & 14.033 \\
\hline $\mathrm{RJ}$ & 3,8 & 3,5 & 3,0 & 2,6 & 2,2 & 2,2 & 1,7 & 1,6 & 1,5 & 1,5 & 1,3 & 1,0 & 0,8 & 0,8 & 0,8 & 0,7 & $-81,4$ & 90.886 \\
\hline SP & 4,1 & 3,1 & 2,9 & 1,2 & 0,8 & 0,6 & 3,6 & 3,4 & 3,1 & 3,2 & 3,4 & 3,2 & 3,2 & 2,8 & 2,1 & 1,9 & $-53,9$ & 198.986 \\
\hline Sul & 4,6 & 3,9 & 3,2 & 2,8 & 2,6 & 2,5 & 2,3 & 2,1 & 1,9 & 1,8 & 1,6 & 1,8 & 1,9 & 2,2 & 2,0 & 1,8 & $-61,6$ & 131.593 \\
\hline PR & 5,3 & 3,5 & 2,5 & 1,8 & 1,8 & 0,8 & 0,9 & 1,6 & 2,0 & 2,0 & 1,8 & 2,0 & 1,7 & 1,9 & 1,7 & 1,2 & $-77,1$ & 47.337 \\
\hline SC & 9,2 & 9,8 & 9,0 & 8,6 & 8,0 & 8,9 & 7,7 & 5,1 & 3,0 & 2,0 & 1,9 & 1,5 & 1,3 & 1,4 & 1,2 & 1,3 & $-86,4$ & 25.418 \\
\hline RS & 2,2 & 1,8 & 1,3 & 1,2 & 1,1 & 1,1 & 1,2 & 1,1 & 1,4 & 1,5 & 1,3 & 1,8 & 2,2 & 2,7 & 2,5 & 2,5 & 16,3 & 58.838 \\
\hline Centro-oeste & 7,2 & 4,8 & 4,1 & 2,9 & 2,8 & 2,5 & 2,6 & 2,8 & 2,7 & 2,1 & 1,8 & 2,2 & 2,0 & 1,9 & 1,8 & 1,7 & $-76,8$ & 49.461 \\
\hline MS & 5,8 & 2,9 & 1,8 & 1,6 & 0,8 & 1,1 & 1,2 & 0,8 & 1,0 & 0,7 & 0,6 & 0,4 & 0,3 & 0,2 & 0,3 & 0,5 & $-92,0$ & 9.694 \\
\hline MT & 2,7 & 2,6 & 1,5 & 0,6 & 0,8 & 0,6 & 0,2 & 0,3 & 0,4 & 0,6 & 0,7 & 0,9 & 1,0 & 1,1 & 1,3 & 0,8 & $-71,8$ & 9.348 \\
\hline $\mathrm{GO}$ & 11,4 & 8,2 & 7,3 & 5,4 & 5,3 & 4,7 & 5,2 & 5,8 & 5,3 & 4,0 & 3,4 & 4,2 & 3,7 & 3,4 & 3,1 & 3,0 & $-74,0$ & 23.296 \\
\hline DF & 3,7 & 1,1 & 1,2 & 1,0 & 1,0 & 0,7 & 0,4 & 0,3 & 0,4 & 0,4 & 0,2 & 0,4 & 0,5 & 0,6 & 0,5 & 0,4 & $-90,2$ & 7.123 \\
\hline Brasil & 8,4 & 7,3 & 6,3 & 5,5 & 5,1 & 4,6 & 5,1 & 4,7 & 4,4 & 4,3 & 3,8 & 3,6 & 3,3 & 3,4 & 3,0 & 2,8 & $-66,3$ & 826.434 \\
\hline Máximo & 28,8 & 27,5 & 24,5 & 24,5 & 22,4 & 21,0 & 20,0 & 17,2 & 15,5 & 14,7 & 13,9 & 10,3 & 8,2 & 8,5 & 8,0 & 8,5 & $-70,5$ & - \\
\hline Mínimo & 1,8 & 1,1 & 0,9 & 0,2 & 0,6 & 0,1 & 0,2 & 0,3 & 0,4 & 0,4 & 0,2 & 0,4 & 0,3 & 0,2 & 0,3 & 0,4 & $-80,5$ & - \\
\hline Amplitude & 26,9 & 26,4 & 23,6 & 24,3 & 21,8 & 20,9 & 19,8 & 17,0 & 15,1 & 14,3 & 13,7 & 9,8 & 8,0 & 8,3 & 7,7 & 8,1 & $-69,8$ & - \\
\hline
\end{tabular}

AC: Acre; AL: Alagoas; AM: Amazonas; AP: Amapá; BA: Bahia; CE: Ceará; DF: Distrito Federal; ES: Espírito Santo; GO: Goiás; MA: Maranhão; MG: Minas Gerais; MS: Mato Grosso do Sul; MT: Mato Grosso; PA: Pará; PB: Paraíba; PE: Pernambuco; PI: Piauí; PR: Paraná; RJ: Rio de Janeiro; RN: Rio Grande do Norte; RR: Roraima: RO: Rondônia; RS: Rio Grande do Sul; SC: Santa Catarina; SE: Sergipe; SP: São Paulo; TO: Tocantins.

Fonte: Sistema de Informações sobre Mortalidade (SIM), 2000-2015 (http://www2.datasus.gov.br/DATASUS/index.php?area=0205). 
Tabela 2

Proporção e número de municípios com preenchimento excelente $(\geq 95 \%)$ da variável raça/cor e número total de municípios segundo regiões, Unidades da Federação (UF) e Brasil, 2000-2015.

\begin{tabular}{|c|c|c|c|c|c|c|c|c|c|}
\hline \multirow[t]{2}{*}{ UF } & \multicolumn{2}{|c|}{2000} & \multicolumn{2}{|c|}{2005} & \multicolumn{2}{|c|}{2010} & \multicolumn{2}{|c|}{2015} & \multirow{2}{*}{$\begin{array}{c}\text { Total de } \\
\text { municípios } \\
\text { (n) }\end{array}$} \\
\hline & $\mathrm{n}$ & $\%$ & $\mathrm{n}$ & $\%$ & $n$ & $\%$ & $n$ & $\%$ & \\
\hline Norte & 254 & 58,7 & 384 & 85,0 & 368 & 81,4 & 380 & 83,7 & 454 \\
\hline $\mathrm{RO}$ & 11 & 22,0 & 33 & 62,3 & 40 & 75,5 & 45 & 84,9 & 53 \\
\hline$A C$ & 3 & 15,8 & 12 & 52,2 & 6 & 27,3 & 17 & 77,3 & 22 \\
\hline AM & 40 & 64,5 & 57 & 90,5 & 58 & 92,1 & 57 & 90,5 & 63 \\
\hline $\mathrm{RR}$ & 11 & 73,3 & 9 & 60,0 & 9 & 60,0 & 12 & 80,0 & 15 \\
\hline PA & 84 & 59,2 & 128 & 88,9 & 132 & 91,7 & 129 & 89,0 & 145 \\
\hline AP & 4 & 33,3 & 16 & 100,0 & 15 & 93,8 & 14 & 87,5 & 16 \\
\hline TO & 101 & 75,9 & 129 & 93,5 & 108 & 77,7 & 106 & 75,7 & 140 \\
\hline Nordeste & 496 & 28,0 & 817 & 45,4 & 954 & 53,0 & 1.068 & 59,3 & 1.802 \\
\hline MA & 88 & 42,7 & 169 & 77,5 & 184 & 84,4 & 185 & 84,9 & 218 \\
\hline $\mathrm{PI}$ & 120 & 55,3 & 172 & 76,8 & 165 & 73,3 & 136 & 60,4 & 225 \\
\hline CE & 45 & 24,3 & 77 & 41,8 & 100 & 54,3 & 144 & 77,8 & 185 \\
\hline $\mathrm{RN}$ & 80 & 48,2 & 57 & 33,9 & 59 & 35,1 & 78 & 46,7 & 167 \\
\hline PB & 55 & 24,9 & 102 & 45,5 & 142 & 63,4 & 132 & 58,9 & 224 \\
\hline PE & 60 & 32,4 & 137 & 74,1 & 134 & 72,0 & 163 & 87,6 & 186 \\
\hline$A L$ & 8 & 7,8 & 8 & 7,8 & 16 & 15,7 & 23 & 22,3 & 103 \\
\hline SE & 4 & 5,3 & 1 & 1,3 & 32 & 42,1 & 33 & 43,4 & 76 \\
\hline BA & 36 & 8,7 & 94 & 22,5 & 122 & 29,2 & 174 & 41,6 & 418 \\
\hline Sudeste & 571 & 34,4 & 977 & 58,4 & 930 & 55,6 & 1.092 & 65,3 & 1.672 \\
\hline MG & 230 & 27,2 & 305 & 35,7 & 413 & 48,4 & 464 & 54,3 & 854 \\
\hline ES & 12 & 15,4 & 13 & 16,5 & 16 & 20,3 & 22 & 27,8 & 79 \\
\hline RJ & 37 & 40,2 & 66 & 71,0 & 79 & 84,9 & 86 & 92,5 & 93 \\
\hline SP & 292 & 45,3 & 593 & 91,8 & 422 & 65,3 & 520 & 80,5 & 646 \\
\hline Sul & 823 & 70,8 & 988 & 83,0 & 1.046 & 87,9 & 1.058 & 88,7 & 1.273 \\
\hline PR & 330 & 82,5 & 387 & 96,8 & 374 & 93,5 & 383 & 95,8 & 400 \\
\hline SC & 109 & 37,1 & 151 & 51,5 & 229 & 77,9 & 254 & 85,8 & 296 \\
\hline RS & 384 & 82,1 & 450 & 90,5 & 443 & 89,3 & 421 & 84,7 & 497 \\
\hline Centro-oeste & 30 & 38,5 & 67 & 84,8 & 73 & 93,6 & 73 & 91,3 & 80 \\
\hline MS & 144 & 32,2 & 327 & 70,0 & 357 & 76,4 & 371 & 78,9 & 390 \\
\hline MT & 71 & 56,3 & 127 & 90,7 & 127 & 89,4 & 132 & 93,0 & 142 \\
\hline GO & 43 & 17,8 & 132 & 53,4 & 156 & 63,4 & 165 & 66,8 & 247 \\
\hline DF & 0 & 0,0 & 1 & 100,0 & 1 & 100,0 & 1 & 100,0 & 1 \\
\hline Brasil & 2.288 & 41,8 & 3.493 & 62,6 & 3.655 & 65,5 & 3.969 & 71,0 & 5.591 \\
\hline
\end{tabular}

AC: Acre; AL: Alagoas; AM: Amazonas; AP: Amapá; BA: Bahia; CE: Ceará; DF: Distrito Federal; ES: Espírito Santo; GO: Goiás; MA: Maranhão; MG: Minas Gerais; MS: Mato Grosso do Sul; MT: Mato Grosso; PA: Pará; PB: Paraíba; PE: Pernambuco; PI: Piauí; PR: Paraná; RJ: Rio de Janeiro; RN: Rio Grande do Norte; RR: Roraima: RO: Rondônia; RS: Rio Grande do Sul; SC: Santa Catarina; SE: Sergipe; SP: São Paulo; TO: Tocantins.

Fonte: Sistema de Informações sobre Mortalidade (SIM), 2015 (http://www2.datasus.gov.br/DATASUS/index. php?area=0205).

Na Região Sudeste, entre 2000 e 2005, houve melhora de completude em todas as UF, com exceção de São Paulo, cuja proporção de municípios com completude excelente passou de 91,8\%, em 2005, para 65,3\%, em 2010. Vale ressaltar que São Paulo tem 646 municípios, o que corresponde a quase 40\% do Sudeste. 
Embora, no geral, tenha se observado melhoras na proporção de municípios com completude excelente, houve significativas pioras em algumas UF no intervalo de tempo estudado. Além de São Paulo, a completude no Acre reduziu de 52,2\% para 27,3\% e Tocantins de 93,5\% para 77,7\% entre 2005 e 2010. Já entre 2010 e 2015, Piauí passou de 73,3\% para 60,4\% e Pernambuco de 63,4\% para 58,9\%.

Apesar da melhora geral na qualidade, a desigualdade no preenchimento excelente da raça/cor ainda pode ser observada em 2015. Enquanto as regiões Nordeste e Sudeste apresentavam 59,3\% e 65,3\% dos municípios com preenchimento excelente, respectivamente, o Norte, Sul e Centro-oeste já apresentavam ao redor de $80 \%$. Desvantagens também foram observadas por UF, onde algumas ainda tinham menos de 50\% de informação excelente: Alagoas (22,3\%), Espírito Santo (27,8\%), Bahia (41,6\%), Sergipe (43,4\%) e Rio Grande do Norte (46,7\%).

Por meio dos resultados do modelo de regressão logística, que tem como desfecho a excelente completude da variável raça/cor no município, percebem-se as características que influenciam a qualidade do preenchimento (Tabela 3). No modelo bruto, a excelente completude mostrou-se positivamente correlacionada com todas as variáveis resposta sobre condição territorial (região e tamanho do município) e socioeconômicas (Índice de Gini, Proporção de Pobreza e IDH municipal). As regiões Sul, Norte e Centro-oeste tiveram mais do dobro de chance de ter excelente completude em relação à Região Nordeste. Municípios pequenos, de IDH baixo ou médio, com alta proporção de pobreza (> $30 \%$ e alta desigualdade (Índice de GINI ruim) tiveram menor chance de ter excelente completude. Vale ressaltar que municípios com pobreza entre 10 e 30\% não foram associados ao desfecho no modelo bruto.

Após ajuste dos fatores no modelo multivariado, foi observado que a região e o tamanho do município são as únicas características que explicaram a excelente qualidade da variável raça/cor (Tabela 3). Municípios das regiões Sul, Norte, Centro-oeste e Sudeste tinham maior chance de ter excelente completude quando comparados com moradores da Região Nordeste $(\mathrm{OR}=6,15 ; 3,80 ; 2,79 ; 1,43$, respectivamente). Os municípios grandes e médios têm cerca de 30\% a mais de chance de ter qualidade excelente da raça/cor que aqueles de pequeno porte (Tabela 3 ).

Foi observada uma mudança na composição dos óbitos de idosos segundo raça/cor no Brasil entre 2000 e 2015 (Figura 1). A população branca no período analisado era a maior parcela da população, entretanto sofreu gradual redução (variação percentual = -13,9\%) no período. A população parda continua a ocupar o segundo lugar na proporção de óbitos de idosos, mas aumentou significativamente (variação percentual $=46,5 \%$ ). Já a população preta, amarela e indígena mantém sua distribuição na população total estável ao longo do período analisado (Figura 1).

Ao comparar a distribuição dos óbitos de idosos segundo raça/cor no SIM com a composição populacional do mesmo grupo no Censo, em 2000 e 2010, foram encontradas diferenças (Tabela 4). $\mathrm{O}$ aumento de pretos e pardos no SIM foi mais acentuado do que o crescimento dessa população no Censo. Apesar disso, no Acre, Rio de Janeiro, Santa Catarina e Rio Grande do Sul, a proporção da população preta e parda aumentou mais entre os Censos do que a proporção de óbitos de idosos pretos e pardos no SIM (Tabela 4).

Em alguns estados, a proporção de óbitos da população preta/parda decresceu, tais como o Amapá, Rio Grande do Norte e Distrito Federal (variação percentual = -22,3\%, -3,8\% e -0,3\%, respectivamente). No Censo, apenas o Piauí apresentou uma ligeira redução da proporção de pretos e pardos (variação percentual $=-0,7 \%$ ). A região na qual se observa maior crescimento dos óbitos de idosos pretos e pardos em relação ao Censo foi o Nordeste, em que o aumento da proporção de óbitos de pretos e pardos chega a ser cinco vezes maior (Razão da variação percentual SIM/Censo $=5,2$ ) (Tabela 4).

\section{Discussão}

Como assinalado na revisão sistemática de Araújo et al. 2, não há consenso sobre a raça/cor como determinante do surgimento de doenças, mas existem robustas evidências das desvantagens da população preta e parda quanto às condições de vida e de saúde 8,9,10,11,13,15. Assim, como explicita o Ministério da Saúde, a boa qualidade da informação no quesito raça/cor é fundamental para identificar agravos, desigualdade e impacto da atenção à saúde 20 . 


\section{Tabela 3}

Chance de completitude excelente da variável raça/cor no município segundo condições de ocorrência do óbito, Brasil, 2015.

\begin{tabular}{|c|c|c|c|c|c|c|c|}
\hline Variáveis/Categorias & $\mathbf{n}$ & OR bruta & Valor de $p$ & IC95\% & OR ajustada & Valor de $p$ & IC95\% \\
\hline \multicolumn{8}{|l|}{ Região } \\
\hline Sul & 1.193 & 5,39 & - & $4,40-6,59$ & 6,15 & 0,00 & $4,62-8,18$ \\
\hline Norte & 454 & 3,53 & - & $2,70-4,61$ & 3,80 & 0,00 & $2,89-4,99$ \\
\hline Centro-oeste & 470 & 2,58 & - & $2,02-3,28$ & 2,79 & 0,00 & $2,08-3,76$ \\
\hline Sudeste & 1.672 & 1,29 & - & $1,13-1,49$ & 1,43 & 0,00 & $1,15-1,78$ \\
\hline Nordeste & 1.802 & 0,00 & & - & 0,00 & & - \\
\hline \multicolumn{8}{|c|}{ Tamanho do município (habitantes) } \\
\hline Grande (> 100.000) & 2.482 & 1,24 & - & $1,07-1,43$ & 1,35 & 0,00 & $1,10-1,65$ \\
\hline Médio (20.001-100.000) & 1.715 & 1,18 & 0,03 & $1,01-1,38$ & 1,33 & 0,00 & $1,12-1,57$ \\
\hline Pequeno $(<20.001)$ & 1.394 & 0,00 & & - & 0,00 & & - \\
\hline \multicolumn{8}{|l|}{ IDH municipal } \\
\hline Alto e muito alto $(>0,699)$ & 2.190 & 1,38 & - & $1,23-1,55$ & 1,09 & 0,26 & $0,94-1,25$ \\
\hline Baixo e médio $(<0,699)$ & 3.401 & 0,00 & & - & 0,00 & & - \\
\hline \multicolumn{8}{|l|}{ Proporção de pobreza (\%) } \\
\hline$<10$ & 2.633 & 1,83 & - & $1,57-2,13$ & 0,83 & 0,21 & $0,62-1,11$ \\
\hline $10-30$ & 1.859 & 1,11 & 0,18 & $0,95-1,30$ & 0,79 & 0,02 & $0,65-0,96$ \\
\hline$>30$ & 2.633 & 0,00 & & - & 0,00 & & - \\
\hline \multicolumn{8}{|l|}{ Índice de Gini } \\
\hline $\operatorname{Bom}(\leq 0,45)$ & 736 & 1,46 & - & $1,20-1,77$ & 0,97 & 0,80 & $0,75-1,24$ \\
\hline Médio $(0,46-0,55)$ & 3.090 & 1,23 & - & $1,08-1,39$ & 1,07 & 0,34 & $0,93-1,24$ \\
\hline Ruim $(\geq 0,56)$ & 1.765 & 0,00 & & - & 0,00 & & - \\
\hline
\end{tabular}

IC95\%: intervalo de 95\% de confiança; IDH: Índice de Desenvolvimento Humano; OR: odds ratio.

Fonte: Sistema de Informações sobre Mortalidade (SIM - http://www2.datasus.gov.br/DATASUS/index.php?area=0205); Estimativas preliminares de população elaboradas pela Secretaria de Vigilância em Saúde/Coordenação-Geral de Informações e Análises Epidemiológicas/Ministério da Saúde; Atlas do Desenvolvimento Humano 2010 (http://atlasbrasil.org.br/2013/pt/o_atlas/quem_faz/atlas_regiao_metropolitana/, accessado em 12/Nov/2018); e Censo Demográfico 2010 (https://censo2010.ibge.gov.br/).

\section{Figura 1}

Distribuição dos óbitos de idosos segundo raça/cor por ano, Brasil 2000-2015

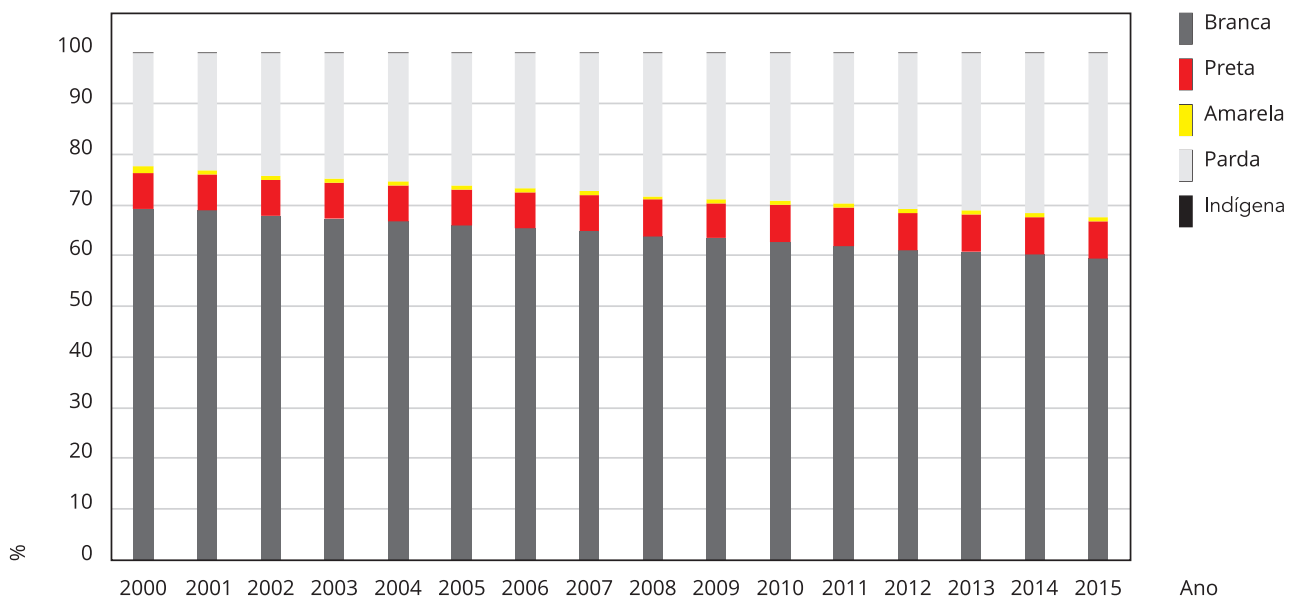

Fonte: Sistema de Informação sobre Mortalidade, 2000 e 2015 (SIM - http://www2.datasus.gov.br/DATASUS/index. php?area=0205). 


\section{Tabela 4}

Proporção de óbitos de idosos pretos e pardos (SIM), proporção de população idosa de pretos e pardos (Censo) e razão da variação percentual entre os anos (SIM/Censo) segundo regiões, Unidades da Federação (UF) e Brasil, 2000 e 2010.

\begin{tabular}{|c|c|c|c|c|c|c|c|}
\hline \multirow[t]{2}{*}{ UF } & \multicolumn{3}{|c|}{ \% óbitos de idosos pretos/pardos (SIM) } & \multicolumn{3}{|c|}{ \% de população idosa preta/parda (Censo) } & \multirow{2}{*}{$\begin{array}{c}\text { Razão da variação } \\
\text { percentual } \\
\text { SIM/Censo }\end{array}$} \\
\hline & 2000 & 2010 & Variação percentual & 2000 & 2010 & Variação percentual & \\
\hline Norte & 67,7 & 72,5 & 7,0 & 67,8 & 70,8 & 4,4 & 1,6 \\
\hline $\mathrm{RO}$ & 43,8 & 53,9 & 23,0 & 51,0 & 58,2 & 14,1 & 1,6 \\
\hline$A C$ & 70,6 & 73,7 & 4,3 & 67,1 & 71,6 & 6,7 & 0,6 \\
\hline AM & 71,9 & 74,3 & 3,3 & 69,5 & 71,6 & 2,9 & 1,1 \\
\hline $\mathrm{RR}$ & 53,4 & 58,8 & 10,0 & 61,7 & 64,4 & 4,4 & 2,3 \\
\hline PA & 71,0 & 76,7 & 8,0 & 71,1 & 73,3 & 3,1 & 2,6 \\
\hline AP & 90,2 & 70,1 & $-22,3$ & 70,7 & 74,2 & 4,9 & $-4,6$ \\
\hline TO & 63,3 & 72,4 & 14,4 & 67,4 & 70,1 & 4,1 & 3,5 \\
\hline Nordeste & 57,9 & 66,1 & 14,1 & 63,7 & 65,4 & 2,7 & 5,2 \\
\hline MA & 66,7 & 75,1 & 12,6 & 71,4 & 74,4 & 4,2 & 3,0 \\
\hline $\mathrm{PI}$ & 70,1 & 75,8 & 8,1 & 72,0 & 71,5 & $-0,7$ & $-12,4$ \\
\hline CE & 53,0 & 65,0 & 22,5 & 60,3 & 63,2 & 4,8 & 4,7 \\
\hline $\mathrm{RN}$ & 49,8 & 47,9 & $-3,8$ & 55,8 & 56,1 & 0,6 & $-6,3$ \\
\hline PB & 49,8 & 62,5 & 25,3 & 55,2 & 56,6 & 2,6 & 9,9 \\
\hline PE & 50,8 & 57,2 & 12,6 & 55,9 & 57,9 & 3,5 & 3,6 \\
\hline$A L$ & 58,5 & 71,0 & 21,4 & 63,4 & 65,1 & 2,6 & 8,1 \\
\hline SE & 55,1 & 68,0 & 23,4 & 66,7 & 67,4 & 1,0 & 22,4 \\
\hline $\mathrm{BA}$ & 68,7 & 73,3 & 6,7 & 69,9 & 71,5 & 2,3 & 2,8 \\
\hline Sudeste & 22,2 & 27,2 & 22,7 & 29,9 & 34,6 & 15,6 & 1,5 \\
\hline MG & 33,2 & 40,0 & 20,5 & 41,2 & 46,6 & 13,2 & 1,6 \\
\hline ES & 38,3 & 41,8 & 9,2 & 42,9 & 46,3 & 8,1 & 1,1 \\
\hline RJ & 32,1 & 35,5 & 10,7 & 37,1 & 41,6 & 12,1 & 0,9 \\
\hline SP & 12,9 & 17,0 & 32,0 & 20,1 & 24,8 & 23,2 & 1,4 \\
\hline Sul & 8,1 & 9,2 & 12,8 & 13,7 & 16,5 & 20,1 & 0,6 \\
\hline PR & 10,4 & 12,9 & 24,5 & 20,2 & 24,7 & 22,5 & 1,1 \\
\hline SC & 4,5 & 4,8 & 7,4 & 8,3 & 11,0 & 33,2 & 0,2 \\
\hline RS & 7,8 & 8,0 & 3,0 & 10,8 & 12,3 & 13,4 & 0,2 \\
\hline Centro-oeste & 40,7 & 47,3 & 16,5 & 44,1 & 49,3 & 11,7 & 1,4 \\
\hline MS & 32,6 & 40,8 & 25,0 & 38,8 & 44,0 & 13,3 & 1,9 \\
\hline MT & 53,1 & 59,0 & 11,2 & 51,8 & 56,0 & 8,0 & 1,4 \\
\hline GO & 38,1 & 47,2 & 23,9 & 43,9 & 49,7 & 13,4 & 1,8 \\
\hline DF & 42,1 & 42,0 & $-0,3$ & 41,8 & 46,2 & 10,5 & 0,0 \\
\hline Brasil & 29,1 & 36,3 & 24,6 & 38,9 & 42,6 & 9,5 & 2,6 \\
\hline
\end{tabular}

AC: Acre; AL: Alagoas; AM: Amazonas; AP: Amapá; BA: Bahia; CE: Ceará; DF: Distrito Federal; ES: Espírito Santo; GO: Goiás; MA: Maranhão; MG: Minas Gerais; MS: Mato Grosso do Sul; MT: Mato Grosso; PA: Pará; PB: Paraíba; PE: Pernambuco; PI: Piauí; PR: Paraná; RJ: Rio de Janeiro; RN: Rio Grande do Norte; RR: Roraima: RO: Rondônia; RS: Rio Grande do Sul; SC: Santa Catarina; SE: Sergipe; SIM: Sistema de Informações sobre Mortalidade; SP: São Paulo; TO: Tocantins.

Fonte: Sistema de Informações sobre Mortalidade (SIM - http://www2.datasus.gov.br/DATASUS/index.php?area=0205); e Censo Demográfico 2000 e 2010 (https://censo2010.ibge.gov.br/).

Acentuada melhora da completude do preenchimento da variável raça/cor na declaração dos óbitos dos idosos desde 2000 foi observada neste trabalho. A média nacional passou de boa para excelente preenchimento até o ano de 2015 (8,4\% e 2,8\%, respectivamente). A partir de 2012, a completude se mantém estável até 2015. Em 2006, a maioria das UF já experimentava completude excelente, inclusive em níveis menores que 1\% de falta de informação (Amapá, Tocantins, Paraná, Mato Grosso e Dis- 
trito Federal), no entanto, no Nordeste, ainda existiam algumas com completude ruim (Sergipe, Bahia, Alagoas). Em 2015, apenas cinco estados não alcançaram qualidade excelente: Alagoas, Bahia, Minas Gerais e Espírito Santo. Ganhos no Brasil sobre qualidade dessa variável a partir do ano 2000 foram também observados para a população geral ${ }^{38}$, para os menores de um ano 14,23 e para abrangências e causas específicas 10,24,27,30.

Nota-se maior amplitude da desigualdade territorial em nível municipal. Em 2015, não apresentaram excelente completude da raça/cor dos óbitos de idosos 1.622 municípios (29\%). Estados da Região Nordeste, assim como o Espírito Santo (Região Sudeste), foram os que tiveram menor proporção de municípios com excelente qualidade (menos de 50\%). Do mesmo modo, Fiorio et al. 24 encontraram grande disparidade da completude da raça/cor entre microrregiões (cerca de 40\%) para todos os óbitos do Espírito Santo no ano de 2005. Pernambuco, segundo Costa \& Frias 26, apesar do incremento observado no período analisado, ainda em 2005, eram poucos os municípios que preenchiam essa variável de maneira satisfatória entre os menores de um ano.

A qualidade da informação da variável raça/cor apresenta significativos diferenciais geográficos e socioeconômicos, quando analisada as chances de um município ter a qualidade de preenchimento excelente. No presente trabalho, observa-se que municípios de IDH baixo ou médio, com alta proporção de pobreza e alta desigualdade tiveram menor chance de ter excelente completude. No entanto, é na condição territorial do município que os diferenciais se manifestam com maior intensidade, como mostrado no modelo ajustado. Municípios pequenos e da Região Nordeste apresentam menores chances de possuir excelência na completude, mesmo quando levado em consideração as demais variáveis. Resultados similares são encontrados no estudo de Andrade \& Szwarcwald 18 acerca da adequação da informação sobre nascimentos e óbitos de menores de um ano, em que se constatou que municípios de pequeno porte possuem pior adequação de informações vitais. As autoras comentam que parece paradoxal que municípios com menor quantidade de eventos vitais tenham pior qualidade, o que pode ser explicado pela iniquidade no acesso a programas e serviços de saúde e pela menor importância dada à informação como evidência da situação local de saúde.

Neste trabalho, mostra-se que a melhora na completude da variável raça/cor dos óbitos de idosos no SIM foi concomitante com o aumento da proporção de óbitos de pretos e pardos. Com isso, a distribuição dos óbitos no SIM por raça/cor torna-se mais parecida com a observada na população idosa recenseada em 2010. Assim, pode-se afirmar que o não preenchimento dessa característica socioeconômica é menos frequente entre a população branca, o que dá indícios de que a desigualdade também atinge a qualidade da informação. Já a tendência ao aumento da proporção de pretos e pardos em todas as fontes de informação observada nas últimas décadas é, em grande parte, impulsionada pela valorização de determinados perfis populacionais étnicos 39 , pelo efeito positivo de políticas públicas inclusivas 40 , além da diferença das taxas de fecundidade segundo raça/cor 41 .

Em estudo que analisou e comparou as taxas de mortalidade infantil por raça/cor a partir do Censo, do SIM e do Sistema de Informações sobre Nascidos Vivos (SINASC) 42, observou-se que, apesar de a taxa ser similar entre as fontes, quando desagregada por raça/cor, foram encontradas amplas diferenças para pretos. A taxa estimada pelo SIM/SINASC foi mais elevada do que pelo Censo. Apesar da possibilidade de que a causa esteja associada ao fato de que, no SIM, a cor do falecido é declarada por pessoa próxima ao morto e, no Censo, ela seja associada à do responsável pelo domicílio, cabe questionar o motivo dessas diferenças atingirem justamente os segmentos mais vulneráveis.

Conforme se evidencia, no presente trabalho, houve uma melhora acentuada no preenchimento da variável raça/cor nas DO dos idosos desde o ano 2000, o que reforça o potencial do SIM para a análise da desigualdade da mortalidade dos idosos e como instrumento para monitoramento, planejamento e gestão sobre equidade no SUS. No entanto, ainda é necessário que a melhora ocorra de forma homogênea em todo o território nacional. Desse modo, revela-se necessário empreender esforços na melhora da completude da raça/cor nos municípios onde ainda não se possui bom preenchimento, visto que a informação é fundamental para uma adequada análise das situações locais de saúde, assim como possibilita a orientação de ações voltadas ao enfrentamento da desigualdade racial na mortalidade de idosos. 


\section{Colaboradores}

Todos os autores fizeram contribuições substanciais para a concepção do trabalho, análise e interpretação dos dados, elaboração e revisão do conteúdo e aprovação final da versão a ser publicada.

\section{Informações adicionais}

ORCID: Dalia Elena Romero (0000-0002-26439797); Leo Maia (0000-0003-1531-0880); Jessica Muzy (0000-0003-2526-2317).

\section{Referências}

1. Barkan S. Sociology: understanding and changing the social world. 2nd Ed. Minneapolis: University of Minnesota Libraries Publishing; 2016.

2. Araújo EM, Costa MCN, Hogan VK, Araújo TM, Dias AB, Oliveira LOA. A utilização da variável raça/cor em Saúde Pública: possibilidades e limites. Interface (Botucatu) 2009; 13:383-94.

3. Pearce N, Foliaki S, Sporle A, Cunningham C. Genetics, race, ethnicity, and health. BMJ 2004; 328:1070-1072.

4. Chehuen Neto JA, Fonseca GM, Brum IV, Santos JLCT, Rodrigues TCGF, Paulino KR, et al. Política Nacional de Saúde Integral da População Negra: implementação, conhecimento e aspectos socioeconômicos sob a perspectiva desse segmento populacional. Ciênc Saúde Colet 2015; 20:1909-16.

5. Marmot M. Social determinants of health inequalities. Lancet 2005; 365:1099-104.

6. Kabad JF, Bastos JL, Santos RV. Raça, cor e etnia em estudos epidemiológicos sobre populações brasileiras: revisão sistemática na base PubMed. Physis (Rio J.) 2012; 22:895-918.

7. Chor D. Desigualdades em saúde no Brasil: é preciso ter raça. Cad Saúde Pública 2013; 29:1272-5.

8. Victora CG, Aquino EM, Carmo Leal M, Monteiro CA, Barros FC, Szwarcwald CL. Maternal and child health in Brazil: progress and challenges. Lancet 2011; 377:1863-76.

9. Reichenheim ME, Souza ER, Moraes CL, Mello Jorge MHP, Silva CMFP, Souza Minayo MC. Violence and injuries in Brazil: the effect, progress made, and challenges ahead. Lancet 2011; 377:1962-75

10. Fiorio NM, Flor LS, Padilha M, Castro DS, Molina MCB. Mortalidade por raça/cor: evidências de desigualdades sociais em Vitória (ES), Brasil. Rev Bras Epidemiol 2011; 14:52230.

11. Batista LE, Escuder MML, Pereira JCR. A cor da morte: causas de óbito segundo características de raça no Estado de São Paulo, 1999 a 2001. Rev Saúde Pública 2004; 38:630-6.

12. Kanso S, Romero DE, Leite IC, Moraes EN. Diferenciais geográficos, socioeconômicos e demográficos da qualidade da informação da causa básica de morte dos idosos no Brasil. Cad Saúde Pública 2011;27:1323-39.
13. Oliveira BLCA, Thomaz EBAF, Silva RA. The association between skin color/race and health indicators in elderly Brazilians: a study based on the Brazilian National Household Sample Survey (2008). Cad Saúde Pública 2014; 30:1438-52.

14. Romero DE, Cunha CB. Avaliação da qualidade das variáveis socioeconômicas e demográficas dos óbitos de crianças menores de um ano registrados no Sistema de Informações sobre Mortalidade do Brasil (1996/2001). Cad Saúde Pública 2006; 22:673-81.

15. Oliveira TC, Medeiros WR, Lima KC. Diferenciais sociodemográficos da mortalidade de idosos em idades precoces e longevas. Rev Baiana Saúde Pública 2015; 18:85-94.

16. Departamento de Monitoramento e Avaliação do SUS, Secretaria Executiva, Ministério da Saúde. Política Nacional de Informação e Informática em Saúde. Brasília: Ministério da Saúde; 2016.

17. Paim J. O que é o SUS. Rio de Janeiro: Editora Fiocruz; 2009. (Coleção Temas em Saúde).

18. Andrade CLT, Szwarcwald CL. Desigualdades sócio-espaciais da adequação das informações de nascimentos e óbitos do Ministério da Saúde, Brasil, 2000-2002. Cad Saúde Pública 2007; 23:1207-16.

19. Batista LE, Monteiro RB, Medeiros RA. Iniquidades raciais e saúde: o ciclo da política de saúde da população negra. Saúde Debate 2013; 37:681-90.

20. Departamento de Análise de Situação de Saúde, Secretaria de Vigilância em Saúde, Ministério da Saúde. Manual de Instruções para o Preenchimento da Declaração de Óbito. Brasília: Ministério da Saúde; 2011. (Série A. Normas e Manuais Técnicos).

21. Departamento de Apoio à Gestão Participativa, Secretaria de Gestão Estratégica e Participativa, Ministério da Saúde. Política Nacional de Saúde Integral da População Negra: uma política do SUS. Brasília: Ministério da Saúde; 2010. (Série B. Textos Básicos de Saúde).

22. Ministério da Saúde. Portaria MS no 344, de 1o de fevereiro de 2017. Dispõe sobre o preenchimento do quesito raça/cor nos formulários dos Sistemas de Informação em Saúde. Diário Oficial da União 2017; 2 fev. 
23. Cardoso AM, Santos RV, Coimbra Jr. CEA. Mortalidade infantil segundo raça/cor no Brasil: o que dizem os sistemas nacionais de informação? Cad Saúde Pública 2005; 21:1602-8.

24. Fiorio NM, Smarzaro DC, Barbosa C, Bisi C. Evolução do preenchimento da variável raça/ cor no sistema de informação sobre mortalidade Vitória/ES de 1996 a 2005. Rev Bras Pesqui Saúde 2008; 10:19-26.

25. Melo GBT, Valongueiro S. Incompletude dos registros de óbitos por causas externas no Sistema de Informações sobre Mortalidade em Pernambuco, Brasil, 2000-2002 e 2008-2010. Epidemiol Serv Saúde 2015; 24:651-60.

26. Costa JMBS, Frias PG. Avaliação da completitude das variáveis da declaração de óbitos de menores de um ano residentes em Pernambuco, 1997-2005. Ciênc Saúde Colet 2011; 16 Suppl 1:1267-74.

27. Rios MA, Anjos KF, Meira SS, Nery AA, Casotti CA. Completude do sistema de informação sobre mortalidade por suicídio em idosos no Estado da Bahia. J Bras Psiquiatr 2013; 62:1318.

28. Messias KLM, Bispo Júnior JP, Pegado MFQ, Oliveira LC, Peixoto TG, Sales MAC, et al. Qualidade da informação dos óbitos por causas externas em Fortaleza, Ceará, Brasil. Ciênc Saúde Colet 2016; 21:1255-67.

29. Felix JD, Zandonade E, Amorim MHC, Castro DS. Avaliação da completude das variáveis epidemiológicas do Sistema de Informação sobre Mortalidade em mulheres com óbitos por câncer de mama na Região Sudeste: Brasil (1998 a 2007). Ciênc Saúde Colet 2012; 17:945-53.

30. Macente LB, Zandonade E. Avaliação da completude do sistema de informação sobre mortalidade por suicídio na Região Sudeste, Brasil, no período de 1996 a 2007. J Bras Psiquiatr 2010; 59:173-81.

31. Carvalho JAM, Rodríguez-Wong LL. A transição da estrutura etária da população brasileira na primeira metade do século XXI. Cad Saúde Pública 2008; 24:597-605.

32. Frias PG, Pereira PMH, Andrade CLT, Szwarcwald CL. Sistema de Informações sobre Mortalidade: estudo de caso em municípios com precariedade dos dados. Cad Saúde Pública 2008; 24:2257-66.

33. Instituto de Pesquisa Econômica Aplicada; ONU Mulheres; Secretaria de Políticas para as Mulheres; Secretaria de Políticas de Promoção da Igualdade Racial. Retrato das desigualdades de gênero e raça. 4a Ed. Brasília: Instituto de Pesquisa Econômica Aplicada; 2011.
34. Programa das Nações Unidas para o Desenvolvimento; Instituto de Pesquisa Econômica Aplicada; Fundação João Pinheiro. Atlas do Desenvolvimento Humano no Brasil. http:// atlasbrasil.org.br/2013/pt/o_atlas/quem_faz/ atlas_regiao_metropolitana/ (acessado em 12/ Nov/2018).

35. Krieger N. Geocoding and monitoring of US socioeconomic inequalities in mortality and cancer incidence: does the choice of areabased measure and geographic level matter?: the Public Health Disparities Geocoding Project. Am J Epidemiol 2002; 156:471-82.

36. Gould JB. Vital records for quality improvement. Pediatrics 1999; 103(1 Suppl E):278-90.

37. Hogan WR, Wagner MM. Accuracy of data in computer-based patient records. J Am Med Inform Assoc 1997; 4:342-55.

38. Braz RM, Oliveira PDTR, Reis AT, Machado NMS. Avaliação da completude da variável raça/cor nos sistemas nacionais de informação em saúde para aferição da equidade étnico-racial em indicadores usados pelo Índice de Desempenho do Sistema Único de Saúde. Saúde Debate 2013; 37:554-62.

39. Carvalho JAM, Wood CH, Andrade D. Notas acerca das categorias de cor dos censos e sobre a classificação subjetiva de cor no Brasil: 1980/90. Rev Bras Estud Popul 2003; 20:2942.

40. Anjos G. A questão "cor" ou "raça" nos censos nacionais. Indicadores Econômicos FEE 2013; 41:103-18.

41. Camarano AA, Kanso S, Carvalho DF, Luz CC. Dinâmica demográfica da população negra brasileira. Comunicado do IPEA 2011; (91). http://www.ipea.gov.br/portal/index.php?op tion $=$ com_content\&view=article \&id $=8391$.

42. Caldas ADR, Santos RV, Borges GM, Valente JG, Portela MC, Marinho GL. Mortalidade infantil segundo cor ou raça com base no Censo Demográfico de 2010 e nos sistemas nacionais de informação em saúde no Brasil. Cad Saúde Pública 2017; 33:e00046516. 


\section{Abstract}

The quality of information on race/color is a necessary condition for knowing the impact of inequality on mortality. This study aims to analyze the trend and inequality in completeness of race/ color in death records of elderly in Brazil's Mortality Information System (SIM in Portuguese) from 2000 to 2015. The study analyzes the completeness of this variable according to different geographic areas, the race or color most affected by poor completeness of records, and the association between excellent completion of race/color and the municipalities' territoriality and socioeconomic status. Data on deaths of elderly individuals were obtained from the SIM and information on the population from censuses and Ministry of Health estimates. The study estimates the percent variation in the proportion of incompleteness. The percent variation of black and brown individuals was estimated from 2000 to 2010 from the SIM and censuses. Crude and adjusted logistic regression $(95 \% \mathrm{CI})$ were used to estimate completeness of race/color as the outcome and territorial and socioeconomic characteristics as independent variables. We found a sharp improvement in quality of completion during the period, especially up to 2006, with an excellent average since 2007. The findings showed territorial inequality at the municipal level. Municipalities with low/medium HDI (with a high proportion of poverty and inequality) showed lower odds of excellent completeness. The adjusted model shows that the region and size of the municipality are characteristics that explain the excellent quality of the race/color variable. Municipalities in Northeast Brazil and small municipalities have the lowest odds of excellent completeness. In conclusion, race/color in the SIM has sufficient quality to be used in studies on inequality of mortality in the elderly, with exceptions at the municipal level.

Aged; Mortality Registries; Data Accuracy; Race Factors; Racism

\section{Resumen}

La calidad de la información sobre raza/color es una condición necesaria para conocer el impacto de la desigualdad en la mortalidad. El objetivo de este trabajo es analizar la tendencia y la desigualdad en la cumplimentación de la raza/color de los óbitos de ancianos en el Sistema de Informacions sobre Mortalidad (SIM) entre 2000 y 2015 en Brasil. Se analiza la cumplimentación de esta variable a través de diferentes ámbitos geográficos, la razal color más afectada por la mala cumplimentación y la asociación entre la excelencia de la cumplimentación de la raza/color con la territorialidad y la condición socioeconómica de los municipios. Los datos de los óbitos de ancianos provienen del SIM y la información de población procede de los censos y estimativas del Ministerio de Salud. Se estima la variación del porcentaje a través de la proporción de la no cumplimentación. La variación del porcentaje de negros/mulatos se estimó entre 2000 y 2010 para el SIM y censos. Se estima regresión logística simple y ajustada (IC95\%), teniendo como desenlace excelente la cumplimentación de la raza/ color y como respuesta variables territoriales y socioeconómicas. Se encontró una acentuada mejora de la calidad de la cumplimentación durante el período, especialmente hasta 2006, siendo excelente la media nacional desde 2007. Se nota desigualdad territorial a nivel municipal. Municipios de IDH bajo/medio, con alta proporción de pobreza $y$ desigualdad, tuvieron una menor oportunidad de contar con una excelente cumplimentación. El modelo ajustado muestra que la región y el tamaño del municipio son las características que explican la excelente calidad de la variable raza/color. Municipios del nordeste y de pequeño porte tienen menos oportunidad de excelente cumplimentación. Se concluye que raza/color en el SIM tiene calidad para ser utilizada en los estudios de desigualdad de la mortalidad de ancianos, salvo excepciones a nivel municipal.

Anciano; Registros de Mortalidad; Exactitud de los Dados; Factores Raciales; Racismo
Recebido em 21/Nov/2018

Versão final reapresentada em 03/Mai/2019

Aprovado em 17/Jun/2019 